\title{
Retos del proceso creativo del diseño a través de la educación online
}

\author{
Aarón Alberto Ruiz Esparza Gutiérrez y Leticia Jacqueline Robles Cuéllar
}

\section{Resumen}

La disciplina del Diseño de Interiores implica el entendimiento de conocimientos y habilidades complejas para su implementación y para el desarrollo idóneo de los procesos de enseñanza y aprendizaje, entre ellos el desarrollo del proceso creativo, que es por antonomasia el proceso de la creatividad, que va de lo abstracto a la invención, construcción y producción del imaginario a la realidad o espacio tangible, manifiesto a través de la generación de proyectos. La docencia de disciplinas relacionadas con el proceso creativo, tradicionalmente llevado a cabo de manera presencial, hoy en día, con la transición a escenarios emergentes de distanciamiento social, o propiamente las nuevas implicaciones de globalización a través de medios virtuales, se promueve la impartición de programas académicos en una modalidad de docencia en línea u online (enseñanza a través del uso de internet), lo cual presenta retos y factores a considerar para que la educación en estas disciplinas de naturaleza prácticas puedan desarrollarse y fomentar el crecimiento intelectual y de creatividad en las formas de instruir y enseñar diseño en los actores de estos procesos de enseñanza disciplinar.

Palabras clave: retos, proceso creativo, diseño, educación en línea.

\section{Introducción}

Ante una modalidad de clases online o de docencia en línea provocada por una situación de contingencia sanitaria por el CoviD-19, es decir, aquella en que los cursos y procesos de aprendizaje se realizan de forma no presencial a través de un dispositivo con conexión a internet, donde se requieren de las Tecnologías de la Información y la Comunicación (TIC) (Dorrego, 2016), surge la siguiente inquietud, ¿se puede llevar a cabo una enseñanza de esta naturaleza en disciplinas como el diseño, específicamente en el diseño de interiores? Hay que considerar que estas disciplinas creativas se insertan en la dimensión humana y que busca interactuar con el otro de forma sensible y social (Ochoa, 2015). El tema presenta de manera general una serie de premisas reflejadas en la educación superior en Diseño de Interiores en modalidad online, donde el centro de atención es el desarrollo del proceso creativo aplicado a un ejercicio práctico, así como la interacción generada, durante el mismo, entre el docente y el estudiante a través de asesorías en línea. Lo anterior en fomento a la reflexión de la comunidad académica sobre los desafíos afrontados durante esta nueva dinámica.

\section{La enseñanza del diseño como proceso creativo}

Los métodos de enseñanza y aprendizaje tradicionales del diseño como disciplina no han sufrido grandes variaciones en los últimos años; sin em- 
bargo, los procesos creativos, ${ }^{1}$ a través de una serie de actos que cumplen con el principio de la doble génesis: lo creado nace primero en nuestra cabeza y luego, por medio de algún proceso mediador, aflora en la realidad (Ferrer, 2006), nos dan la pauta para plantear una solución fundamentada, satisfactoria y actual a cualquier proyecto de diseño.

En una metodología pedagógica de diseño, los procesos creativos tradicionalmente conciben la interacción entre el profesor y el estudiante como entes extensivos de las herramientas que se utilizan para la presentación, la comprensión y la creación de un proyecto de diseño, el cual se manifiesta a partir de un discurso teórico (que surge por medio de una investigación) y que se refleja de forma material a través de la representación física o digital, con el propósito final de expresar una posible solución (fundamentada a través del briefing ${ }^{2}$ ) a una problemática de diseño (Perdomo, 2019).

En este proceso de interacción pedagógica, encontramos características como el intercambio de información verbal y no verbal, tanto de forma directa como indirecta, no sólo es responsable el alumno, sino también el profesor (López y Alfonso, 2018), quien a través de la retroalimentación y asesoría guía el proceso creativo, tratando de definir con éxito un intercambio de ideas, derivando en interpretaciones de diseño propuestas por el alumno y su reflexión personal planteando estrategias para resolver las necesidades de un problema cuya lectura sea clara para otros y que concluye en la representación material y gráfica de este proceso creativo.

Bajo estas premisas, entonces, ¿realmente se puede desarrollar un proceso creativo bajo el esquema de una educación en línea? Para abordar esta cuestión es necesario entender cómo se gesta la participación entre el alumno y el profesor, tener conciencia de los rasgos del lenguaje comunicacional en el que se expresa el diseño y cómo se desarrolla el proceso creativo de aprendizaje.

1 Es una capacidad expresiva al resolver problemas, encontrando para ello los medios adecuados que sirvan a las soluciones que se tienen en mente.

2 Briefing: es la relación de necesidades puntuales y requerimientos indispensables, tangibles e intangibles que presenta un problema a solucionar, los cuales se investigan y analizan para poder establecer una contrapropuesta o posible solución (Academia de Diseño de Interiores, 2020).
《En una metodología pedagógica de diseño los

procesos creativos tradicionalmente conciben la

interacción entre el profesor y el estudiante como

entes extensivos de las herramientas que se

utilizan para la presentación, la comprensión y la

creación de un proyecto \)

\section{Educación del diseño online}

Tomando en cuenta que en la modalidad de educación online los alumnos no necesitan asistir presencialmente al espacio físico de estudio y que, de cierta forma, promueve una solución al problema de masividad, se deberán considerar una serie de premisas con las que el alumno lidiará debido a que generalmente su interacción tendrá lugar en grupos virtuales, pero trabajará en soledad, como lo menciona Cárdenas (2014), el estado del arte actual plantea analogías y diferencias entre la modalidad presencial y online, lo que sugiere una serie de replanteamientos epistémicos en la relación de enseñanza y aprendizaje, manifestando que una enseñanza homologada o adecuada a la educación en línea aún se encuentra en desarrollo.

El surgimiento y uso de herramientas digitales de apoyo para el desarrollo del Diseño de Interiores, como Sketchup, Revit, Lumion, FloorPlaner, Room Planner, Canvas, entre otros (Monti1la, 2019), y el fácil acceso a material informático de esta índole, se han manifestado como una opción al momento de la creación de proyectos, en un entorno de aprendizaje la responsabilidad en el manejo adecuado y la calidad. En el uso de este material será una parte fundamental en los pasos metodológicos planteados para el desarrollo creativo del estudiante, reflejado en los alcances y sus resultados; con esto surge el desafío de considerar una variación en el enfoque de la docencia fomentando el desarrollo creativo con énfasis donde el alumno sea capaz de construir su propio aprendizaje siendo así "el eje central y protagonista principal de su quehacer" (UAA, 2007, p. 7), como se manifiesta en el Modelo Educativo Institucional (MEI) de la Universidad Autónoma de Aguascalientes. 


\section{Formación en la práctica online}

En un entorno pedagógico presencial, aula o taller, se toma en cuenta que el desarrollo creativo se puede manifestar desde dos vertientes: la primera parte de las instrucciones, métodos y puntos de vista del docente hacia el alumno, para que éste pueda entender aspectos figurativos o abstractos; la segunda va en una dirección inversa, en la cual el alumno, a partir de su trabajo interpretativo, muestra al docente posibles soluciones a la problemática de diseño planteada, sin embargo, este proceso es cíclico, consecutivamente se genera un intercambio de información hasta que se logran resultados que satisfacen de forma creativa las necesidades que demande la naturaleza de cada proyecto de diseño.

Comprendiendo que el estudiante está en el proceso de desarrollo de un criterio de pensamiento sensible y analítico propio, que acorde al MEI "lo conducirá a la obtención [...] y dominio de información según su formación profesional" (UAA, 2007, p. 8), habrá de enfatizarse en ese desarrollo orientado hacia la creatividad, el compromiso y la apertura en el intercambio de opiniones, basado en la observación reflexiva, misma que "no es una habilidad natural que el estudiante posee (o no). [...] [Lo anterior] implica realizar experiencias orientadas al desarrollo de esa habilidad en el aula de diseño" (Terrile, 2010).

\section{Desarrollo creativo en ejercicio práctico de diseño vía online}

En este apartado se ejemplifican los procesos previamente mencionados, representados a través de imágenes tomadas de los avances paulatinos de un proyecto, resultante de la interacción entre el alumno y el profesor, en las asesorías online, (debido a la contingencia sanitaria) dentro de la ma-

《El estado del arte actual plantea analogías y diferencias entre la modalidad presencial y online,

lo que sugiere una serie de replanteamientos epistémicos en la relación de enseñanza y aprendizaje manifestando que, una enseñanza homologada o adecuada a la educación en línea, aún se encuentra en desarrollo \》 teria de Taller de Fundamentos del Diseño II, de $2^{\circ}$ semestre de la carrera de Diseño de Interiores -es en ese semestre en el cual los estudiantes tienen su primer acercamiento al diseño tridimensional después de pasar por un semestre adquiriendo conceptos de composición abstracta-.

La Figura 1 representa uno de los primeros esbozos e ideas generales que, por medio de croquis y esquemas, buscan dar soluciones creativas al diseño de una secuencia de espacios a nivel abstracto, partiendo siempre de instrucciones y lineamientos por parte del profesor y planeación del ejercicio; si bien esta etapa fue representada por medios manuales, el desarrollo y la interacción entre el asesor y alumno, se establece en diferentes formas y sesiones de comunicación sincrónica y asincrónica con la finalidad de favorecer el proceso creativo de la estudiante.

Figura 1. Croquis conceptuales

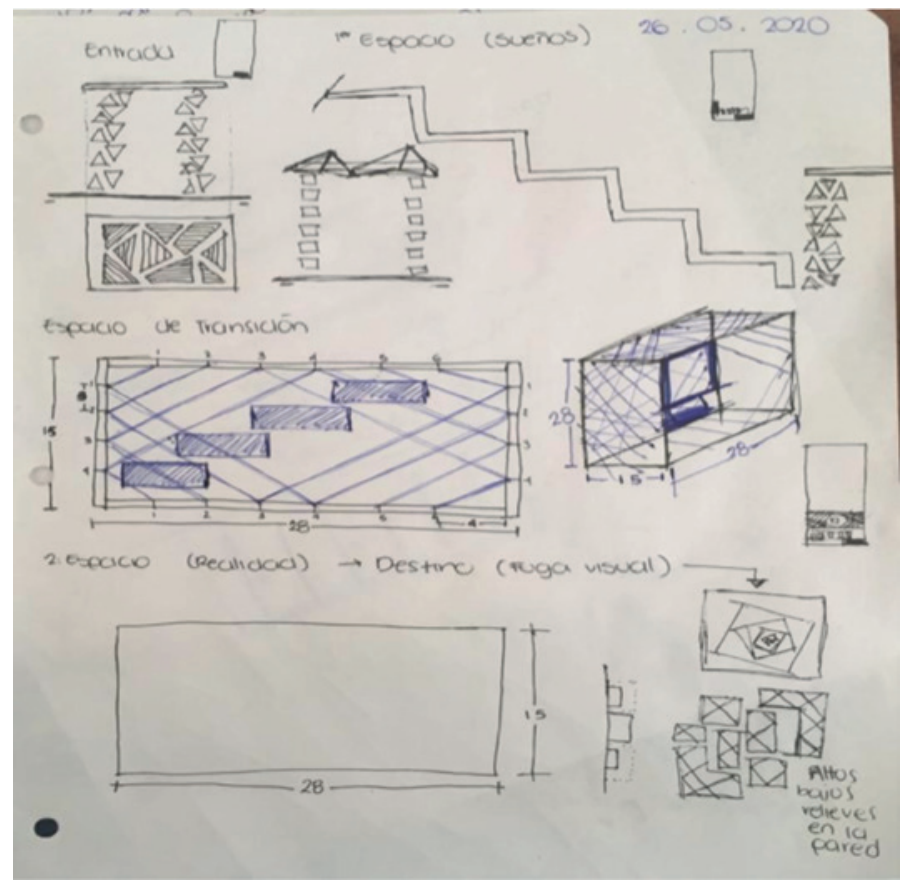

Fuente: Esquemas conceptuales derivados de los lineamientos del proyecto trazados por Jessica G. Vázquez Enciso (2020), estudiante del $2^{\circ}$ semestre de la licenciatura en Diseño de Interiores en el Taller de Fundamentos del Diseño II. 
Figura 2. Maqueta de hipótesis con evolución de conceptos

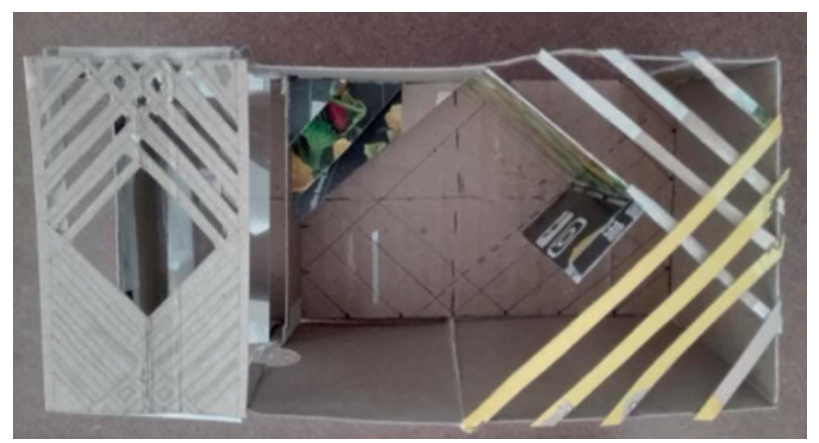

Fuente: Maqueta de hipótesis con evolución de conceptos derivados de asesorías online, cumpliendo los parámetros del proyecto de diseño. Elaborada por Jessica G. Vázquez Enciso (2020), estudiante del $2^{\circ}$ semestre de la licenciatura en Diseño de Interiores, en el Taller de Fundamentos del Diseño II.

Figura 3. Maqueta final

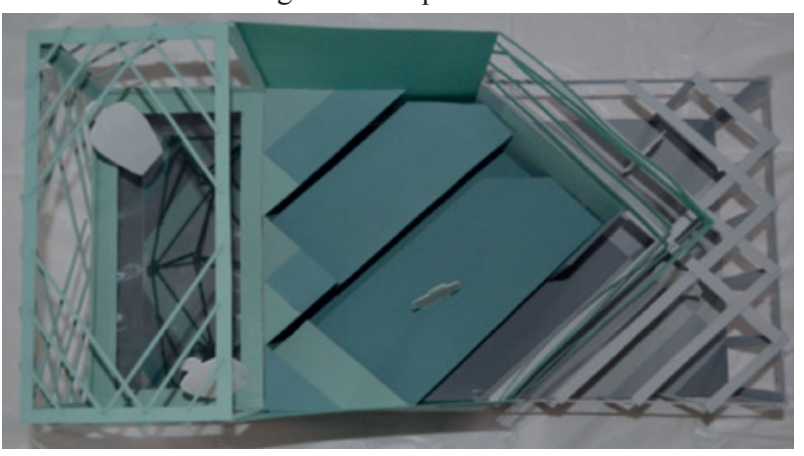

Fuente: Maqueta que representa los parámetros solicitados para la elaboración de un recorrido que conecta espacios de forma abstracta, integrando elementos de composición de diseño básico. Elaborada por Jessica G. Vázquez Enciso (2020), estudiante del $2^{\circ}$ semestre de la licenciatura en Diseño de Interiores, en el Taller de Fundamentos del Diseño II.

Subsecuentemente, en la Figura 2 se representa la traducción material (física) por medio de una hipótesis de solución a los conceptos solicitados para el proyecto, resaltando cambios significativos consecuencia de la toma de decisiones del propio aprendizaje de la estudiante. Finalmente, en la Figura 3 se muestra la propuesta final donde las TIC permitieron que la interacción y el desarrollo del proceso creativo se pudiera generar mediante el intercambio de ideas entre el profesor y los alumnos, derivado del compromiso y la buena comunicación entre ambos, concluyendo en la expresión gráfica y material del proyecto.
«En la modalidad online, la interacción

alumno-profesor se promueve también a través

del diseño de instrucciones y actividades

sincrónicas y asincrónicas, considerando

el tiempo necesario para su desarrollo,

a fin de evitar el posible desgaste físico,

cognitivo y emocional de los participantes,

y su falta de atención y compromiso \》

\section{Conclusiones}

Si bien el proceso creativo de diseño en disciplinas prácticas se puede desarrollar, en las escuelas es donde se "puede hacer una pausa por un minuto para pensar en la experiencia de aprendizaje que desean crear" (Villafuerte, 2020) y así enfrentar una serie de retos factibles de sortear para su reflexión y atención en una modalidad online como se presenta a continuación:

1. Sobre la interacción alumno-profesor

a. Fomentar siempre una comunicación clara entre los participantes con actitud de apertura, considerando que en los intercambios de ideas de las propuestas de diseño pueden presentar diferentes interpretaciones cognitivas por parte de profesores y estudiantes.

b. En el diseño de instrucciones y actividades sincrónicas y asincrónicas, considerar el tiempo necesario y oportuno para su desarrollo acorde a la naturaleza de la materia, a fin de evitar el desgaste físico, cognitivo y emocional de los participantes, así como la falta de atención y compromiso por parte de los mismos.

2. Respecto a las Tecnologías de la Información y la Comunicación

a. Considerar que puede existir una falta de accesibilidad y experiencia en el uso de las TIC, o bien que, en algunos niveles, los participantes son neófitos en el uso de las mismas, creando así una brecha en los procesos de enseñanza y aprendizaje. 
«Para llevar a cabo la evaluación de los

aprendizajes en la educación online, es

recomendable el uso -de manera sincrónica y

asincrónica- de los Instrumentos Académicos para

la Evaluación de Aprendizajes: desarrollados

por la academia de Diseño de Interiores de la

UAA, para medir valores cuantitativos y cualitativos

en diferentes áreas de un proyecto como: la

viabilidad de diseño, presentación, representación,

y el desempeño del estudiante, entre otros 》

3. De la evaluación de los aprendizajes

a. Implementar de manera sincrónica y/o asincrónica el uso de instrumentos, herramientas didácticas y estrategias de medición como los Instrumentos Académicos para la Evaluación de Aprendizajes
(IAPEA), ${ }^{3}$ estas herramientas deben estar acorde con los niveles de aprendizaje de los alumnos.

4. Sobre los docentes

a. Un replanteamiento pedagógico que permeé sobre lo administrativo en la designación de actividades y cargas semestrales para que los docentes no sólo sean guías en el desarrollo intelectual y creativo de los estudiantes, sino que realmente se considere el tiempo y la capacitación necesaria para ser creadores de contenido digital, asesores, retroalimentadores, evaluadores y analistas de los trabajos derivados de cada materia.

3 EI IAPEA es una herramienta desarrollada por la academia de Diseño de Interiores de la UAA para medir valores cuantitativos y cualitativos en diferentes áreas de un proyecto como la viabilidad de diseño, presentación y representación, desempeño del estudiante, entre otros.

\section{Fuentes de consulta}

Cárdenas, I. (2014). Enseñar y aprender diseño en la virtualidad, Actas de Diseño No. 16. vIII. Encuentro Latinoamericano de Diseño "Diseño en Palermo" Cuarto Congreso Latinoamericano de Enseñanza del Diseño, Buenos Aires, Argentina.

Dorrego, E. (2016). Educación a distancia y evaluación del aprendizaje. RED. Revista de Educación a Distancia, 50(12), 1-20. Universidad Central de Venezuela. Recuperado de http://www.um.es/ead/ $\mathrm{red} / 50 /$ dorrego.pdf.

Ferrer, E. (2006). El proceso creativo. Grupo Ferrer-ITAM. Recuperado de http://segmento.itam.mx/Administrador/Uploader/material/El\%20Proceso\%20Creativo.PDF.

López, C. y Alfonso A. (2018). La comunicación educativa en la educación a distancia. EDUCREA. Recuperado de https://educrea.cl/la-comunicacion-educativa-en-la-educacion-a-distancia/.

Montilla, A. (2019). Diseño de Interiores online: herramientas útiles. Revista digital INESEM. Recuperado de https://revistadigital.inesem.es/diseno-y-artes-graficas/herramientas-diseno-interiores/.

Ochoa, E. (2015). El diseño como disciplina eminentemente práctica, transformativa y comunicativa. Iconofacto, 11(17), 74-80.

Perdomo, S. (2019). Conoce las fases del proceso creativo antes de diseñar. DEUSTO Formación. Recuperado de https://www.deustoformacion.com/blog/diseno-produccion-audiovisual/conoce-fasesproceso-creativo-antes-disenar.

Terrile, A. (2010) ¿Se puede enseñar diseño a distancia? America Learning \& Media. [Blog]. Recuperado de http://www.americalearningmedia.com/edicion-004/49-innovacion/205-ise-puede-ensenardiseno-a-distancia.

UAA. (2007). Modelo Educativo Institucional. Correo Universitario, séptima época, (15). [Primera reimpresión], 29 de mayo de 2015. México: UAA. Recuperado de https://bit.ly/2TPzsUo.

Villafuerte, P. (4 de mayo de 2020). El aprendizaje remoto enfrenta otro reto: el profesorado no está preparado para la enseñanza en línea. [Blog]. Observatorio de innovación educativa TEC. Recuperado de https://observatorio.tec.mx/edu-news/profesorado-no-esta-preparado-para-educacion-online. 